\title{
Nonlinear Vibration Analysis of Helical Face-Gear Transmission System with Multi-Factor
}

\author{
Liu Bo, Zhao Jiangang, Qian Jianhua and Wang Xiaoyu \\ China Nuclear Power Technology Research Institute, 518031 Shenzhen, China.
}

\begin{abstract}
A systematic analysis of the dynamics of a helical face-gear system with 8 degree of freedom is performed in this study under complex excitation. The nonlinear dynamic system is solved by the Runge-Kutta method. The bifurcation and dynamic load characteristics of the system is identified from a series of diagrams. The effect of multi-factor on bifurcation diagrams is also analysed. The results real that with the increase of dimensionless frequency, the system undergoes the process of periodic, chaotic and periodic motions. The amplitude of the dynamic load gradually increases in a certain range and begins to decrease at a certain value. A higher mesh damping coefficient or a higher input torque or a lower gear backlash can reduce the vibration or eliminate chaotic responses.
\end{abstract}

\section{Introduction}

Face gear transmission has the characteristics of light weight, simple structure, high contact ratio and low meshing noise. The transmission has found application in helicopter transmissions [1] and other important aviation transmission device widely. Some domestic and foreign experts [2-6] have done the related research work in the principle of gear engagement, tooth surface contact strength, tooth root bending strength, tooth profile machining and profile modification. Due to the gear transmission system includes parameters of gear backlashes [7], time-varying mesh stiffness [8], mesh damping [9], transmission errors [10], tooth surface friction [11], the system is a strongly nonlinear system and the vibration properties of the gear system will directly affect the stability and reliability of the system [12]. In the nonlinear dynamics of gear system, the author [13] did some research relevantly and analyzed the effect of speed ratios, backlashes and load on the dynamic response of gear system. Specially, scholars [14-15] have done some research on the nonlinear vibration properties of the face-gear system, but most of them not fully consider the influence of multi-factor on the gear system. Meanwhile, studies on the nonlinear vibration behavior of the helical face-gear system is very rare at present.

In this research, a bending-torsion-axial coupled nonlinear dynamic model of helical face-gear with 8 degrees of freedom is established, including the factors of time-varying mesh stiffness, mesh damping, support stiffness, support damping, transmission errors, backlashes, tooth surface friction and external excitation. Fourth-fifth order Runge-Kutta method with variable time step is introduced to solve the dimensionless dynamic differential equations. The bifurcation and dynamic load characteristic of the system is identified from the bifurcation diagrams, the time histories, phase diagrams, Poincaré maps and the diagram of dynamic load coefficient using the dimensionless frequency as control parameters. The effect of multi-parameter on bifurcation diagrams is also analyzed.

\section{System model}

According to the principle of gear engagement, the model of orthogonal helical face-gear can be built shown in Fig. 1. Rectangular coordinate system is established at the two gear axis intersection point. $x$-axis coincides with the axis of the face-gear, the $y$-axis coincides with the axis of the helical pinion, tangential line of the gear is parallel to $z$ axis.

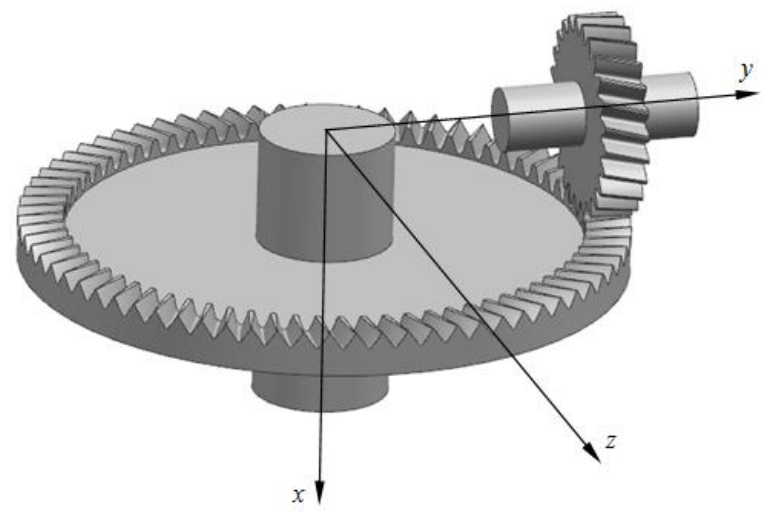

Figure 1. Model of orthogonal helical face-gear

According to the feature of engagement, the axial force in $y$ direction, the tangential force in $z$ direction, and 
radial force in $x$ direction are applied to the helical pinion. Radial force in $y$ direction, tangential force in $z$ direction and axial force in $x$ direction are applied to the face-gear. The elastic supported dynamic model of the helical facegear transmission system is built under those forces, as is shown in Fig. 2. In the model, $k_{I j}(I=X, Y, Z, j=\mathrm{p}, \mathrm{g})$ is the bearing stiffness; $c_{I j}(I=X, Y, Z, j=\mathrm{p}, \mathrm{g})$ is the bearing damping; $b_{I j}(I=X, Y, Z, j=\mathrm{p}, \mathrm{g})$ is the bearing backlash; $K_{h}$ is the mesh stiffness; $c_{h}$ is the mesh damping; $f$ is the nonlinear friction coefficient; $b_{h}$ is the gear backlash; $e(t)$ is the transmission errors and varies as a function of time. Both gears are simulated with concentrated mass and inertia, gear shafts are regarded as rigid body, and the bearing stiffness and damping for both ends of the shafts along the $x, y, z$-direction are treated equivalently.

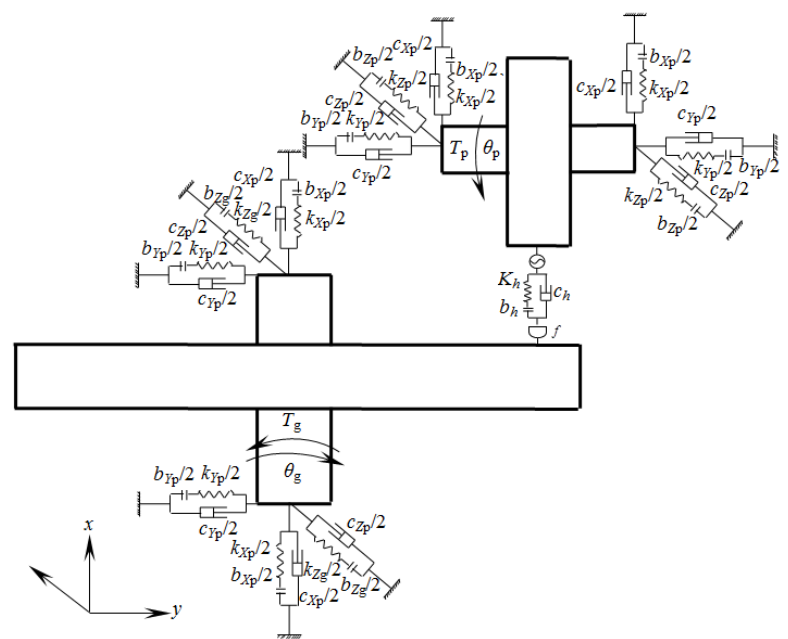

Figure 2. Dynamic model of orthogonal helical face-gear drive system

The translational vibration freedom $\left(X_{\mathrm{p}}, Y_{\mathrm{p}}, \mathrm{Z}_{\mathrm{p}}, X_{\mathrm{g}}, Y_{\mathrm{g}}\right.$, $\left.Z_{\mathrm{g}}\right)$ along $x, y, z$-direction and degrees of freedom in rotation around the body axial direction $\left(\theta_{\mathrm{p}}, \theta_{\mathrm{g}}\right)$ are considered respectively. Then, there are 8 DOF in the gear system, expressed as

$$
\left\{X_{\mathrm{p}}, Y_{\mathrm{p}}, Z_{\mathrm{p}}, \theta_{\mathrm{p}}, X_{\mathrm{g}}, Y_{\mathrm{g}}, Z_{\mathrm{g}}, \theta_{\mathrm{g}}\right\}^{\mathrm{T}}
$$

\section{Nonlinear dynamic formulation}

Assuming the following parameters

$$
\left\{\begin{array}{l}
a_{1}=\sin \alpha_{n} \sin \beta \\
a_{2}=\sin \alpha_{n} \cos \beta \\
a_{3}=\cos \alpha_{n} \sin \beta \\
a_{4}=\cos \alpha_{n} \cos \beta \\
a_{5}=\sin \alpha_{n} \\
a_{6}=\cos \alpha_{n}
\end{array}\right.
$$

in which $\alpha_{n}$ is the pressure angle. $\beta$ is the helical angle.

Due to the vibration displacement and the transmission errors, the gear pair produces the relative displacement along the normal direction of the meshing point, which can be written as

$$
\begin{aligned}
\lambda_{n}= & a_{4}\left(r_{\mathrm{p}} \theta_{\mathrm{p}}-r_{\mathrm{g}} \theta_{\mathrm{g}}\right)+a_{4}\left(Z_{\mathrm{p}}-Z_{\mathrm{g}}\right)+a_{3}\left(Y_{\mathrm{p}}-Y_{\mathrm{g}}\right) \\
& +a_{5}\left(X_{\mathrm{p}}-X_{\mathrm{g}}\right)-e(t)
\end{aligned}
$$

where $r_{\mathrm{p}}$ is the pitch radius of the pinion, $r_{\mathrm{g}}$ is the nominal radius of the face-gear, $r_{\mathrm{g}}=\mathrm{m} z_{\mathrm{g}} / 2 . \theta_{\mathrm{p}}$ and $\theta_{\mathrm{g}}$ are the angle of the pinion and the face-gear. $e(t)$ is the transmission errors induced by the gear machining and installation errors and can be written as

$$
e(t)=e_{0}+e_{r} \cos \left(\omega_{h} t+\phi_{r}\right)
$$

in which $e_{0}$ and $e_{r}$ are the constant and the variable of transmission errors, $\omega_{h}$ is the mesh frequency, $\phi_{r}$ is the initial phase angle.

The dynamic mesh force along the normal direction can be performed as

$$
F_{n}=K_{h}(t) f\left(\lambda_{n}\right)+c_{h} \dot{\lambda}_{n}
$$

in which $c_{h}$ is the mesh damping. $\lambda_{n}$ is the relative displacement along the normal direction of the meshing point. $f\left(\lambda_{n}\right)$ is the backlash function and varies as a function of time. $K_{h}(t)$ is the time-varying mesh stiffness which is a cycle of periodic function for meshing period and can be expressed as

$$
K_{h}(t)=K_{m}+K_{r} \cos \left(\omega_{h} t+\phi_{r}\right)
$$

in which $K_{m}$ is the mean mesh stiffness, $K_{r}$ is the variable amplitude of mesh stiffness.

The coordinate axis component of the dynamic mesh force can be performed as

$$
\left\{\begin{array}{l}
F_{x}=a_{5} F_{n}+a_{6} \eta f F_{n} \\
F_{y}=a_{3} F_{n}-a_{1} \eta f F_{n} \\
F_{z}=a_{4} F_{n}-a_{2} \eta f F_{n}
\end{array}\right.
$$

in which $f$ is the friction coefficient. $\eta$ is the direction coefficient of the friction force which is determined by the nominal sliding velocity and can be expressed as

$$
\eta=\operatorname{sign}\left(l_{p}-r_{b 1} \tan \alpha_{n}\right)
$$

in which $r_{b 1}$ is the base cylinder radius of the pinion. $l_{p}$ is the friction arm of tooth on any point of the actual line of contact which can be expressed as

$$
l_{p}=\sqrt{r_{a 1}^{2}-r_{b 1}^{2}}-\varepsilon P_{b 1}+\omega_{1} r_{b 1} t
$$

in which $\varepsilon$ is the contact ratio of the gear pair, $\omega_{1}$ is the rotational speed of the pinion, $r_{\mathrm{a} 1}$ is the addendum cylinder radius of the pinion, $P_{b 1}$ is the base pitch of the pinion.

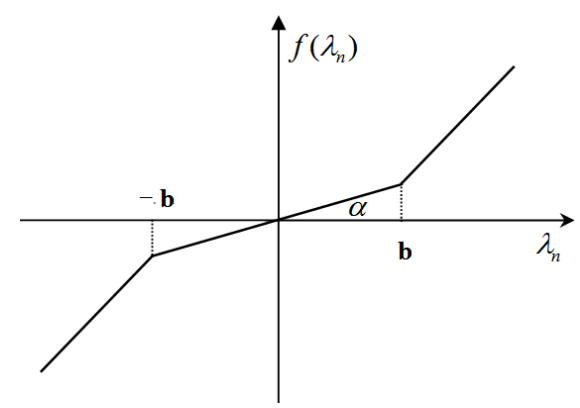

Figure 3. Backlash function diagram 
The tooth backlash is one of the important factors that causes the nonlinear vibration of the gear system and is a strongly nonlinear term in the gear system dynamic equations. The effect of backlash is considered as a displacement type nonlinear function shown in Fig. 3 and described as follows

$$
f\left(\lambda_{n}\right)=\left\{\begin{array}{cc}
\lambda_{n}-b_{h}(1-\alpha) & \lambda_{n}>b_{h} \\
b_{h} \alpha & \left|\lambda_{n}\right| \leq b_{h} \\
\lambda_{n}+b_{h}(1-\alpha) & \lambda_{n}<-b_{h}
\end{array}\right.
$$

in which $b_{h}$ is the half of the total gear backlash, $\alpha$ is the stiffness ratio between the two contact stages.

The bearing supporting shaft has a certain gap and also uses the same backlash nonlinear function as the gears to express. Bearing supporting force along $x, y, z$ coordinate direction can be expressed as follows

$$
F_{I j}=k_{I j} f\left(I_{j}\right)
$$

in which $I_{j}$ and $k_{I j}(I=X, Y, Z, j=\mathrm{p}, \mathrm{g})$ are the bearing backlash and stiffness along the $x, y, z$-direction for different gears.

According to the Newton's Second Law, the equations of the helical face-gear transmission system dynamic model under the assumption of nonlinear suspension can be written as

$$
\left\{\begin{array}{l}
m_{\mathrm{p}} \ddot{X}_{\mathrm{p}}+c_{X \mathrm{p}} \dot{X}_{\mathrm{p}}+k_{X \mathrm{p}} f\left(X_{\mathrm{p}}\right)=-F_{x} \\
m_{\mathrm{p}} \ddot{Y}_{\mathrm{p}}+c_{Y \mathrm{p}} \dot{Y}_{\mathrm{p}}+k_{Y \mathrm{p}} f\left(Y_{\mathrm{p}}\right)=-F_{y} \\
m_{\mathrm{p}} \ddot{Z}_{\mathrm{p}}+c_{Z \mathrm{p}} \dot{Z}_{\mathrm{p}}+k_{Z \mathrm{p}} f\left(Z_{\mathrm{p}}\right)=-F_{z} \\
J_{\mathrm{p}} \ddot{\theta}_{\mathrm{p}}=T_{\mathrm{p}}-F_{z} r_{\mathrm{p}} \\
m_{\mathrm{g}} \ddot{X}_{\mathrm{g}}+c_{X \mathrm{~g}} \dot{X}_{\mathrm{g}}+k_{X \mathrm{~g}} f\left(X_{\mathrm{g}}\right)=F_{x} \\
m_{\mathrm{g}} \ddot{Y}_{\mathrm{g}}+c_{Y \mathrm{~g}} \dot{Y}_{\mathrm{g}}+k_{Y \mathrm{~g}} f\left(Y_{\mathrm{g}}\right)=F_{y} \\
m_{\mathrm{g}} \ddot{Z}_{\mathrm{g}}+c_{Z \mathrm{~g}} \dot{Z}_{\mathrm{g}}+k_{Z \mathrm{~g}} f\left(Z_{\mathrm{g}}\right)=F_{z} \\
J_{\mathrm{g}} \ddot{\theta}_{\mathrm{g}}=-T_{\mathrm{g}}+F_{z} r_{\mathrm{g}}
\end{array}\right.
$$

in which $T_{\mathrm{p}}$ and $T_{\mathrm{g}}$ are the input torque and output torque which can be expressed as

$$
\left\{\begin{array}{l}
T_{p}=T_{0}+T_{\mathrm{e} 1} \sin \omega_{1} t \\
T_{g}=-T_{0}+T_{\mathrm{e} 2} \sin \omega_{2} t
\end{array}\right.
$$

in which $T_{0}$ is the mean torque, $\omega_{1}$ and $\omega_{2}$ are the rotational speed of the pinion and the face-gear. $T_{e 1}$ and $T_{e 2}$ are the variable amplitude of the input and the output excitation torque.

Taking the relative displacement along the normal direction of the meshing point $\lambda_{n}$ as a new degree of freedom and merging the torsional vibration equations of Eq.(12), we can get

$$
\begin{gathered}
m_{e} \ddot{\lambda}_{n}-a_{5} m_{e} \ddot{X}_{\mathrm{p}}+a_{5} m_{e} \ddot{X}_{\mathrm{g}}-a_{3} m_{e} \ddot{Y}_{\mathrm{p}}+a_{3} m_{e} \ddot{Y}_{g} \\
-a_{4} m_{e} \ddot{Z}_{\mathrm{p}}+a_{4} m_{e} \ddot{Z}_{\mathrm{g}}+a_{4}^{2} c_{h} \dot{\lambda}_{n}+a_{4}^{2} K_{h}(t) f\left(\lambda_{n}\right) \\
=F_{\mathrm{p}}+F_{\mathrm{g}}+F_{\mathrm{p} f}+F_{\mathrm{g} f}-m_{e} \ddot{e}(t)
\end{gathered}
$$

In which $m_{e}, F_{\mathrm{p}}, F_{\mathrm{p} f}, F_{\mathrm{g}}$ and $F_{\mathrm{g} f}$ can be performed as

$$
\left\{\begin{array}{l}
m_{e}=J_{\mathrm{p}} J_{\mathrm{g}} /\left(r_{\mathrm{p}}^{2} J_{\mathrm{g}}+r_{\mathrm{g}}^{2} J_{\mathrm{p}}\right) \\
F_{\mathrm{p}}=a_{4} m_{e} r_{\mathrm{p}} T_{\mathrm{p}} / J_{\mathrm{p}} \\
F_{\mathrm{p} f}=a_{2} a_{4} m_{e} r_{\mathrm{p}} l_{\mathrm{p}} \eta f F_{n} / J_{\mathrm{p}} \\
F_{\mathrm{g}}=a_{4} m_{e} r_{\mathrm{g}} T_{\mathrm{g}} / J_{\mathrm{g}} \\
F_{\mathrm{g} f}=a_{2} a_{4} m_{e} r_{\mathrm{g}}^{2} \eta f F_{n} / J_{\mathrm{g}}
\end{array}\right.
$$

in which $J_{\mathrm{p}}$ and $J_{\mathrm{g}}$ are the moment of inertia of the pinion and the face-gear.

Introducing the dimensionless parameters, Eqs. (12) and (14) can be expressed as

$$
\left\{\begin{array}{l}
\ddot{x}_{\mathrm{p}}+2 \xi_{x \mathrm{p}} \dot{x}_{\mathrm{p}}+\left(a_{5}+a_{6} \eta f\right) \xi_{h \mathrm{p}} \dot{\lambda}+k_{x \mathrm{p}} f\left(X_{\mathrm{p}}\right) \\
+\left(a_{5}+a_{6} \eta f\right) k_{h \mathrm{p}} f(\lambda)=0 \\
\ddot{y}_{\mathrm{p}}+2 \xi_{\mathrm{yp}} \dot{y}_{\mathrm{p}}+\left(a_{3}-a_{1} \eta f\right) \xi_{h \mathrm{p}} \dot{\lambda}+k_{y \mathrm{p}} f\left(Y_{\mathrm{p}}\right) \\
+\left(a_{3}-a_{1} \eta f\right) k_{h \mathrm{p}} f(\lambda)=0 \\
\ddot{z}_{\mathrm{p}}+2 \xi_{z \mathrm{p}} \dot{z}_{\mathrm{p}}+\left(a_{4}-a_{2} \eta f\right) \xi_{h \mathrm{p}} \dot{\lambda}+k_{z \mathrm{p}} f\left(Z_{\mathrm{p}}\right) \\
+\left(a_{4}-a_{2} \eta f\right) k_{h \mathrm{p}} f(\lambda)=0 \\
\ddot{x}_{\mathrm{g}}+2 \xi_{x \mathrm{~g}} \dot{x}_{\mathrm{g}}-\left(a_{5}+a_{6} \eta f\right) \xi_{h \mathrm{~g}} \dot{\lambda}+k_{x \mathrm{~g}} f\left(X_{\mathrm{g}}\right) \\
-\left(a_{5}+a_{6} \eta f\right) k_{h \mathrm{~g}} f(\lambda)=0 \\
\ddot{y}_{\mathrm{g}}+2 \xi_{\mathrm{gg}} \dot{y}_{\mathrm{g}}-\left(a_{3}-a_{1} \eta f\right) \xi_{h \mathrm{~g}} \dot{\lambda}+k_{y \mathrm{~g}} f\left(Y_{\mathrm{g}}\right) \\
-\left(a_{3}-a_{1} \eta f\right) k_{h \mathrm{~g}} f(\lambda)=0 \\
\ddot{z}_{\mathrm{g}}+2 \xi_{z \mathrm{~g}} \dot{z}_{\mathrm{g}}-\left(a_{4}-a_{2} \eta f\right) \xi_{h \mathrm{~g}} \dot{\lambda}+k_{z \mathrm{~g}} f\left(Z_{\mathrm{g}}\right) \\
-\left(a_{4}-a_{2} \eta f\right) k_{h \mathrm{~g}} f(\lambda)=0 \\
\ddot{\lambda}_{-} a_{5} \ddot{x}_{\mathrm{p}}+a_{5} \ddot{x}_{\mathrm{g}}-a_{3} \ddot{y}_{\mathrm{p}}+a_{3} \ddot{y}_{\mathrm{g}}-a_{4} \ddot{z}_{\mathrm{p}}+a_{4} \ddot{z}_{\mathrm{g}} \\
+a_{4}^{2} \xi_{h} \dot{\lambda}_{+}+a_{4}^{2} k_{h} f(\lambda)=f_{\mathrm{p}}+f_{\mathrm{g}}+f_{\mathrm{p} f}+f_{\mathrm{g} f}-f_{e}
\end{array}\right.
$$

in which the dimensionless parameters can written as

$$
\begin{aligned}
& x_{j}=X_{j} / b_{h} \quad, \quad y_{j}=Y_{j} / b_{h} \quad, \quad z_{j}=Z_{j} / b_{h} \\
& \lambda=\lambda_{n} / b_{h}, \omega_{n}=\sqrt{K_{m} / m_{e}}, \omega_{i j}=\sqrt{k_{I j} / m_{j}} \text {, } \\
& \xi_{i j}=c_{I j} /\left(2 m_{j} \omega_{n}\right), \xi_{h j}=c_{h} /\left(2 m_{j} \omega_{n}\right), \omega=\omega_{h} / \omega_{n}, \\
& \xi_{h}=c_{h} /\left(2 m_{e} \omega_{n}\right), \tau=\omega_{n} t, f_{j}=F_{j} /\left(m_{e} b_{h} \omega_{n}^{2}\right), \\
& f_{j f}=F_{j f} /\left(m_{e} b_{h} \omega_{n}^{2}\right), k_{h}=1+\frac{K_{r}}{m_{e} \omega_{n}^{2}} \cos \left(\omega \tau+\phi_{r}\right), \\
& f_{e}=\frac{e_{r} \omega^{2}}{b_{h}} \cos \left(\omega \tau+\phi_{r}\right), k_{i j}=\omega_{i j}^{2} / \omega_{n}^{2}, \\
& k_{h j}=K_{h}(\tau) /\left(m_{j} \omega_{n}^{2}\right), f(\lambda)=\left\{\begin{array}{cc}
\lambda+\alpha-1 & \lambda>1 \\
\alpha & |\lambda| \leq 1 \\
\lambda+\alpha+1 & \lambda<-1
\end{array}\right. \\
& f\left(I_{j}\right)=\left\{\begin{array}{cc}
I_{j} / b_{h}-(1-\alpha) b_{i j} / b_{h} & I_{j}>b_{i j} / b_{h} \\
\alpha & \left|I_{j}\right| \leq b_{i j} / b_{h} \\
I_{j} / b_{h}+(1-\alpha) b_{i j} / b_{h} & I_{j}<-b_{i j} / b_{h}
\end{array}\right. \\
& I=X, Y, Z, i=x, y, z, j=\mathrm{p}, \mathrm{g} .
\end{aligned}
$$




\section{Numerical simulation and discussion}

The nonlinear dynamic equations presented in Eqs. (16) for the helical face-gear system were solved by the Fourth-fifth order Runge-Kutta method with variable time step. The time step in the iterative solution procedure was assigned a value of $T_{m} / 300\left(T_{m}=2 \pi / \omega, T_{m}\right.$ is the dimensionless mesh cycle) and the iterative time was set to $300 T_{m}$. The first hundreds of cycles were specifically excluded to ensure the analyzed data related to steady-state conditions. The chaotic motion of the system was identified by the bifurcation diagrams, time history, phase diagrams and Poincaré maps. The dynamic load characteristic of the system was analyzed by the diagram of dynamic load coefficient which was variation with time.

\subsection{Bifurcation diagram}

The bifurcation diagram for the dimensionless displacement of helical face-gear system using the dimensionless frequency $\omega$ as control parameter at gear backlash $\left(b_{h}=1\right)$, meshing damping coefficient $\left(c_{h}=0.14\right)$, input torque $\left(T_{0}=850 \mathrm{~N} \cdot \mathrm{m}\right)$ is shown in Fig. 4 . It can be obtained that the system displays single periodic motion at the low values of the dimensionless frequency, i.e. $\omega<0.6$. Especially, there exist the jump phenomenon in the diagram at $\omega=0.5$. As $\omega$ is increased from 0.6 to 0.9 , the single-periodic is replaced by $n \mathrm{~T}$-periodic and the system state changes from 2T-periodic motion $(\omega=0.6-$ $0.8)$ to $4 \mathrm{~T}$-periodic motion $(\omega=0.8-0.9)$. For values of the dimensionless frequency in the range $\omega=0.9$ to 0.96 , the system performs chaotic motion. As $\omega$ is further increased from $\omega=0.96$ to 1.05 , the chaotic motion transits to $n \mathrm{~T}$-periodic motion. However, at higher values of the dimensionless frequency $(\omega=1.05-1.75)$, the gear system undergoes chaotic motion once again. Finally, for all $\omega>1.75$, the chaotic motion is replaced by $2 \mathrm{~T}$-periodic and single periodic motion.

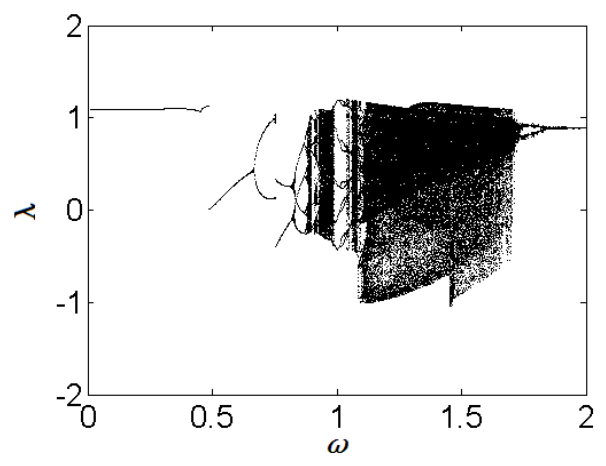

Figure 4. Bifurcation diagram using dimensionless frequency, $\omega$, as control parameter.

\subsection{Prediction of the dynamic responses}

To observe the response and chaotic behavior of the helical face-gear system at some values of the dimensionless frequency, the time history, phase diagrams and Poincaré maps of the gear system as shown in Figs. 5-12 are draw at $\omega=0.3,0.7,0.85,0.95,1.0,1.4$,
1.8 and 1.9, respectively. Significantly, when the phase diagrams form a closed curve, the Poincaré maps show $n$ discrete points and the time history curves are highly regular, the system is in periodic motion, i.e. $\omega=0.3,0.7$, $0.85,1.8$ and 1.9. On the contrary, the phase diagrams is highly disordered, the Poincaré maps form geometrically fractal structures and the time history curves become irregular, the system exhibits a chaotic behavior, i.e. $\omega=0.95$ and 1.4. However, at the values of the dimensionless frequency $\omega=0.3$ and 1.9 , the displacement amplitude is significantly smaller than others, which are in regular single periodic motion.
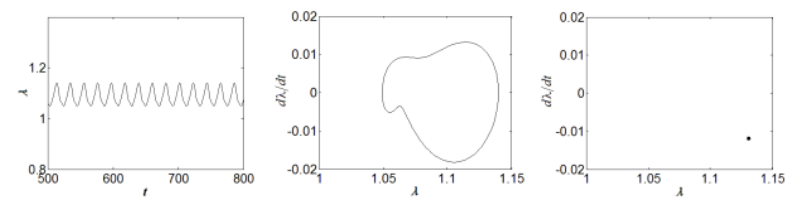

Figure 5. Simulation results with dimensionless frequency $\omega=0.3$.
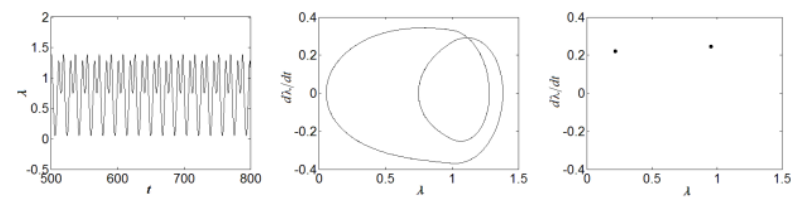

Figure 6. Simulation results with dimensionless frequency $\omega=0.7$.
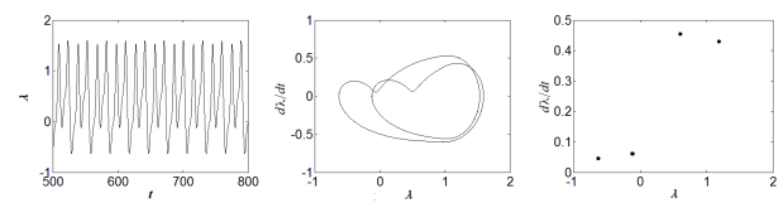

Figure 7. Simulation results with dimensionless frequency $\omega=0.85$.
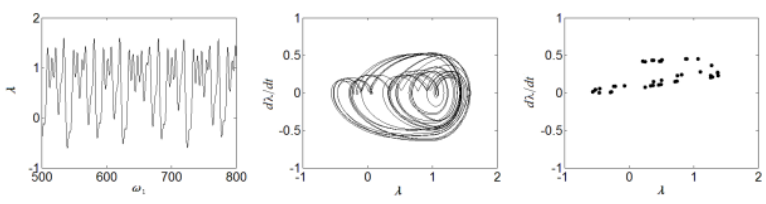

Figure 8. Simulation results with dimensionless frequency $\omega=0.95$.
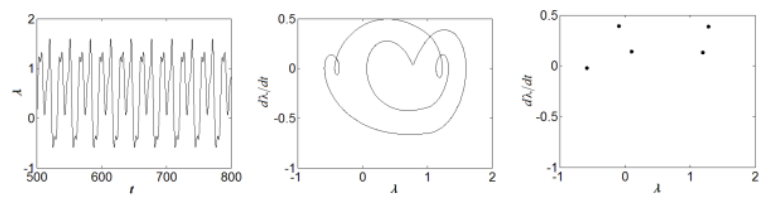

Figure 9. Simulation results with dimensionless frequency $\omega=1.0$.
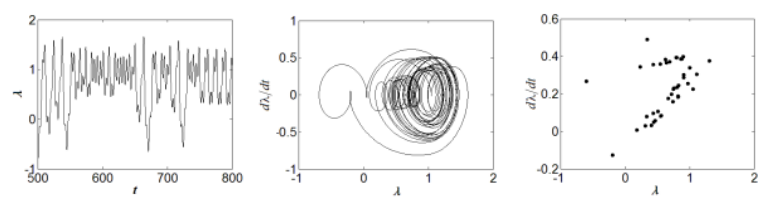

Figure 10. Simulation results with dimensionless frequency $\omega=1.4$. 


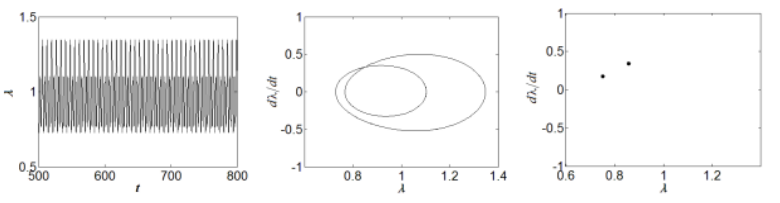

Figure 11. Simulation results with dimensionless frequency $\omega=1.8$.
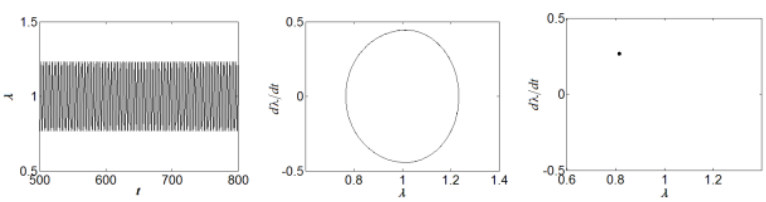

Figure 12. Simulation results with dimensionless frequency $\omega=1.9$.

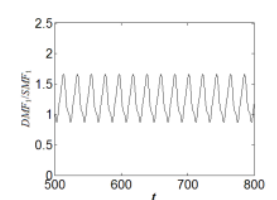

(a) $\omega=0.3$

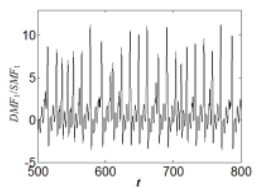

(d) $\omega=1.2$

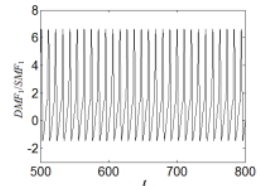

(b) $\omega=0.6$

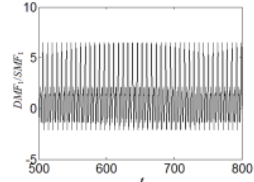

(e) $\omega=1.5$

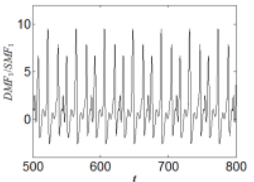

(c) $\omega=0.9$

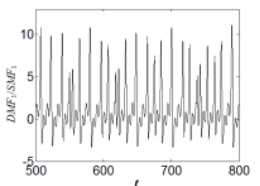

(f) $\omega=1.8$
Figure 13. Dynamic load coefficient of the system with different dimensionless frequency, $\omega$.

Furthermore, to observe the dynamic load characteristic of the system, Fig. 13 illustrates the corresponding diagram of dynamic load coefficient $\left(D M F_{1} / S M F_{1}\right)$ at different values of the dimensionless frequency. In the range of $\omega=0.3-1.5$, the $D M F_{1} / S M F_{1}$ becomes larger, as the $\omega$ is increased. However, at the high value of the $\omega=1.8$, the $D M F_{1} / S M F_{1}$ is significantly small. Corresponding to the time history diagrams, the trend of time history diagrams is consistent with the trend of the dynamic load coefficient. In other words, choosing the suitable speed ratio can decrease the dynamic response and load of the gear system.

\subsection{Effect of multi-parameter on bifurcation diagrams}

Figs. 14-16 illustrate the corresponding bifurcation diagram of the system at different dimensionless meshing damping coefficients, gear backlashes and input torques. With the increase of the dimensionless meshing damping coefficient $c_{h}$ and input torque $T_{0}$, the Chaos areas of the bifurcation diagrams become smaller gradually, which means that the system trends to be more stable. However, with the increase of the dimensionless gear backlash $b_{h}$, the chaos areas and the displacement amplitude become larger obviously. The motion of the system trends to be more complicated. So, the dynamic response of the system can be improved with a higher mesh damping coefficient or a higher input torque or a lower gear backlash. All in all, the bifurcation diagram of the gear system can be controlled by selecting right parameters.

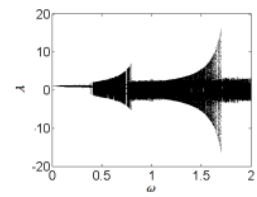

(a) $c_{\mathrm{h}}=0.001$

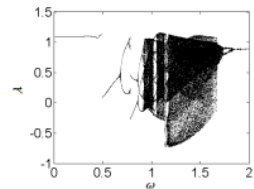

(d) $c_{\mathrm{h}}=0.16$

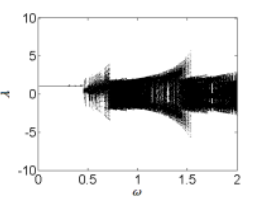

(b) $c_{\mathrm{h}}=0.01$

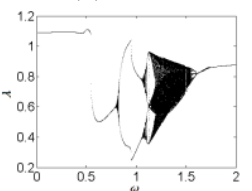

(e) $c_{\mathrm{h}}=0.32$

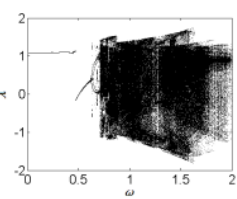

(c) $c_{\mathrm{h}}=0.07$

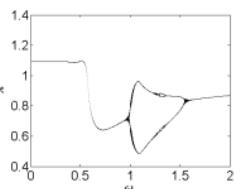

(f) $c_{\mathrm{h}}=0.46$
Figure 14. Bifurcation diagram using dimensionless frequency, $\omega$, as bifurcation parameter at different dimensionless meshing damping coefficients, $c_{h}$.

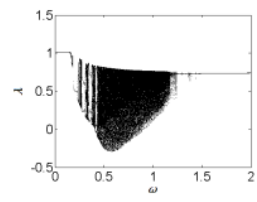

(a) $T_{0}=50 \mathrm{~N} \cdot \mathrm{m}$

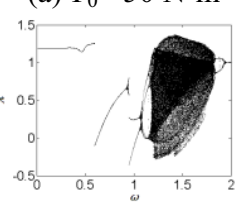

(d) $T_{0}=2000 \mathrm{~N} \cdot \mathrm{m}$

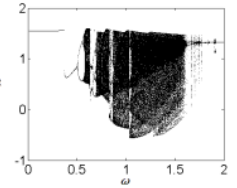

(b) $T_{0}=500 \mathrm{~N} \cdot \mathrm{m}$

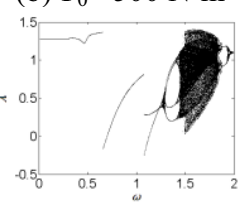

(e) $T_{0}=2000 \mathrm{~N} \cdot \mathrm{m}$

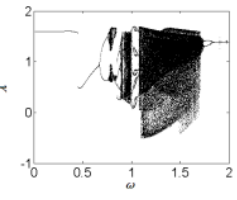

(c) $T_{0}=1000 \mathrm{~N} \cdot \mathrm{m}$

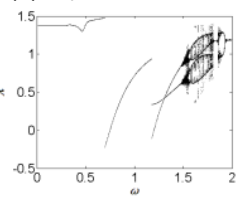

(f) $T_{0}=2500 \mathrm{~N} \cdot \mathrm{m}$
Figure 15. Bifurcation diagram using dimensionless frequency, $\omega$, as bifurcation parameter at different input torques, $T_{0}$.

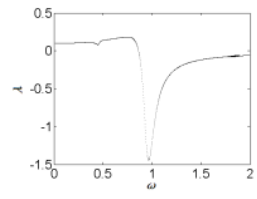

(a) $b_{h}=0.01$

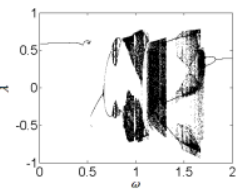

(d) $b_{h}=0.5$

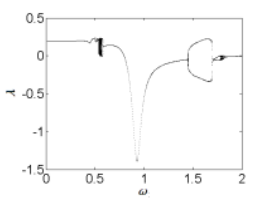

(b) $b_{h}=0.1$

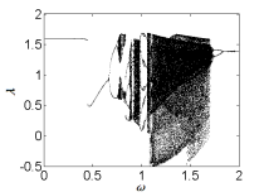

(e) $b_{h}=1.5$

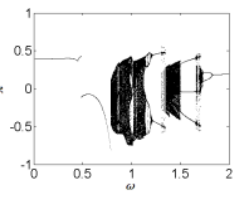

(c) $b_{h}=0.3$

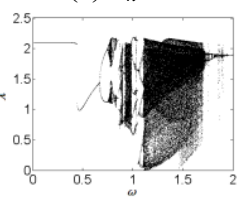

(f) $b_{h}=2$
Figure 16. Bifurcation diagram using dimensionless frequency, $\omega$, as bifurcation parameter at different dimensionless gear backlash, $b_{h}$.

\section{Conclusions}

In this study, the dynamic characteristics of a helical face-gear system with 8 degree of freedom have been investigated under considerations of the time-varying mesh stiffness, mesh damping, support stiffness, support damping, transmission errors, backlashes, tooth surface friction and external excitation. The chaotic motion and dynamic load characteristics of the system have been discussed by the bifurcation diagrams, time history, phase diagrams Poincaré maps and the diagram of dynamic load coefficient. The study has been analyzed the bifurcation 
diagrams of the system using dimensionless frequency as bifurcation parameter at different dimensionless mesh damping coefficients, gear backlashes and input torques, respectively.

(i) With the increase of dimensionless frequency, the system undergoes the process of periodic, chaotic and periodic motions. Jumping phenomenon and double Chaos areas can be found in the bifurcation diagram.

(ii) The amplitude of the dynamic response and load is small, when the system is in the regular periodic motion. So, choosing the suitable speed ratio can decrease the vibration or avoid chaotic motion.

(iii) With the increase of the dimensionless mesh damping coefficient or input torque, the system trends to be more stable. With the increase of the dimensionless gear backlash, the system becomes more complicated. So, the dynamic response of the system can be improved with a higher mesh damping coefficient or a higher input torque or a lower gear backlash.

The last, the systematic analysis of the chaotic and dynamic load characteristics of the helical face-gear nonlinear dynamic system under multi-factor conditions can offer a method to decrease the dynamic response and load of the gear system.

\section{References}

1. Litvin F L, Wang J C, Bossler R B, J. Mech. Des. N. Y 116, 672-676 (1992)
2. Litvin F L, Zhang Y, Wang J C, J. Mech. Des. N. Y 114, 642-647 (1992)

3. Litvin F L, Gonzalez-Perez I, Fuentes A, Comput. Method. Appl. M 194, 3870-3901(2005)

4. Tsay M F, Fong Z H. P, I. MECH. ENG. C-J. MEC 221, 715-725(2007)

5. Zhao N, Guo H, Fang Z D, C. J. A. P 23, 21422146(2008)

6. Cui Y M, Fang Z D, Su J Z, C. J. A 23, 13461351(2013)

7. Chen S Y, Tang J Y, Luo C, MECH. MACH. THEORY 46, 466-478(2011)

8. He S, Gunda R, Singh R, J, SOUND. VIB 301, 929949(2007)

9. Chang-Jian C W, Chang S M, NONLINERA ANALREAL 12, 979-989(2011)

10. Sun $\mathrm{T}, \mathrm{Hu} \mathrm{H} \mathrm{Y}, \mathrm{MECH}$. MACH. THEORY 38, 1371-1390(2003)

11. Vaishya M, Singh R. J, SOUND. VIB 348, 671694(2001)

12. Lewichki D G, Heath G F, Filler R R.A. F. P, AHSInter 2, 1051-1060(2007)

13. Lin T J, Wang D H, Ran X T, J. V. S. 32, 1-7(2013)

14. Yang Z, Wang S M, Fan Y S, J. C. U 34, 26-35(2011)

15. Chen S Y, Tang J Y, Chen W T, MEC 49, 10231037(2014) 